\title{
Stability Analysis for Seed Cotton Yield and Its Components in Upland Cotton (Gossypium hirsutum L.)
}

\author{
Pinki", S.S. Siwach, R.S. Sangwan, Sombir Singh, V.S. Mor, \\ Shiwani Mandhania, Sunayana and Neha Rohila \\ CCS Haryana Agricultural University, Hisar, Haryana, India \\ *Corresponding author
}

\section{A B S T R A C T}

\section{Keywords}

Cotton, stability analysis, Seed cotton yield, Component traits,

Additive effects

Article Info

Accepted:

12 March 2018

Available Online:

10 April 2018
The present study was conducted on stability analysis for seed cotton yield and its component traits in three varieties H 1098 - I, H 1300 and H 1316 under six environments (three sowing periods i.e. early, normal and late sown conditions in year 2015 \& 2016). Observations were recorded for days to first flower, Days to bursting, Plant height $(\mathrm{cm})$, Number of monopods /plant, Number of bolls/ plants, Boll weight (g), Number of seeds/boll, Ginning out turn (\%), Lint index (g), Seed index (g), Lint yield/ plant (g) and Seed cotton yield/ plant (g). The analysis of variance computed for the twelve characters in six environments revealed that mean square due to genotypes were highly significant for all the traits. Mean squares due to environment (linear) and linear interaction genotypes $\times$ environment were significantly for most of the traits studied. After comparing the different environments of a particular year, it clearly showed that early sowing was most favorable, followed by normal sowing and late sowing as it resulted in poorest performance for most of the traits.

\section{Introduction}

Cotton, G. hirsutum is grown in India under a wide range of climatic conditions. India has a pride place in the global cotton scenario due to several distinct features such as the largest area under cotton (105 lakh ha) representing about one-third of the global cotton area (330 lakh ha) with production of $560 \mathrm{~kg} / \mathrm{ha}$ in the world (ICAR 2016-17). In crop performance, three factors are very important i.e. genotype, the environment in which the crop is grown, and the interactions between genotype and environment $(\mathrm{G} \times \mathrm{E})$ (Gomez, Gomez, 1984). The agro ecological diversity of environments complicates breeding and testing of improved genotypes with adequate adaptation, but it also permits identification of extreme environmental conditions that guarantee selection pressure from important stresses.

Estimation of phenotypic stability has proven to be a valuable tool in the assessment of varietals adaptability. It is generally agreed that, the more stable genotypes can somehow adjust their phenotypic responses to provide some measures of uniformity in spite of environmental fluctuations. More knowledge about causes of $\mathrm{G} \times \mathrm{E}$ interaction is needed and would be useful for establishing breeding 
objectives, identifying the best test condition and finding areas of optimal cultivar adaptation. This information can be obtained by description of individual genotype performance in various environments because it allows identification of genotypic traits involved in $\mathrm{G} \times \mathrm{E}$ interaction. In the presence of significant GE interactions, stability parameters are estimated to determine the superiority of individual genotypes across the range of environments. Genotype $\times$ location, genotype $x$ year and genotype $\times$ location $\times$ year interaction components were found to be significant for seed cotton yield in past studies (Campbell et al., 2012; Killi and Harem, 2006). The present study was carried out to determine the effect, of $\mathrm{G} \times \mathrm{E}$ interaction on the yields and yield components traits.

\section{Materials and Methods}

The experiment was conducted during kharif 2015 \& 2016 having three cultivars H 1098-I, $\mathrm{H} 1300$ and $\mathrm{H} 1316$ of American cotton grown at CCS Haryana Agricultural University, Hisar in randomized block design replicated six times each in eight rows of $6 \mathrm{~m}$ length with a spacing of $67.5 \times 30 \mathrm{~cm}$. These varieties were grown in six environments that comprises of three sowings periods (Early: first fortnight of April, normal: first fortnight of May and late: end of May/early June) during the year 2015 $\& 2016$.

Observations were recorded on five competitive plants excluding border plants in each cultivar from each replication in each environment. Data were recorded for the traits viz; days to first flower, plant height $(\mathrm{cm})$, number of monopods/plant, number of effective bolls per plant, boll weight $(\mathrm{g})$, number of seeds per boll, ginning out turn $(\%)$, lint index $(\mathrm{g})$, seed index $(\mathrm{g})$, seed cotton yield per plant (g), lint yield/plant $(\mathrm{g})$. Stability analysis was carried out by Eberhart and Russell method (1966).

\section{Results and Discussion}

The analysis of variance computed for the twelve characters in six environments (Table 1) revealed that mean square due to genotypes were highly significant for all the traits over all environments indicating presence of variability among the genotypes and environments. All the morphological traits such as days to first flower, days to boll bursting, plant height, number of monopods /plant, number of bolls /plant, number of bolls /plant, number of seeds/boll, ginning out turn $(\%)$, seed index $(\mathrm{g})$, lint index $(\mathrm{g})$, lint yield / plant and seed cotton yield were high in early sown condition as compared to normal and late sown conditions because in early sown condition a plant had more time to develop properly and utilize the existing environmental factors as well as nutrition from soil also. Similar pattern of results for days to first flower, plant height, number of monopods / plant, bolls / plant, ginning outturn, seed index, lint index, lint yield/plant and seed cotton yield /plant were reported by Dahiphale et al., 2018. The mean performance of genotypes along with their range for different environments under study is given in table 2 . A brief description of the results is given here under.

\section{Days to first flower}

The overall range for this character in the six environments in the variety $\mathrm{H} 1098$ - I was 53.6 - 69.8, H 1300 was 50 - 64.5 and for the variety H 1316 was 49.2 - 70.1.The mean number of days of all the three varieties to flower was 68.13, 60.73, 50.93, 68.13, 60.37and 53.37 in environments $E_{1}$ to $E_{6}$ respectively.

\section{Days to bursting}

The overall range for this character in the six environments for the variety H 1098- I was 
89.2 - 110.3, H 1300 was 93.5 - 112.9 and for the variety $\mathrm{H} 1316$ was $90.7-110.6$. The mean numbers of days to boll bursting of all the three varieties were $111.27,102.57,91.13$, 112.27, 109.27 and 106.13 in environments $E_{1}$ to $\mathrm{E}_{6}$ respectively

\section{Plant height (cm)}

The overall range for this character in the six environments for the variety $\mathrm{H}$ 1098- I was122.7 - 164.8, H 1300 was 111.7 - 160.9 and for the variety H 1316 was 116.6 - 185.7 . The mean plant height of all the three varieties was $160.57,146.57,117,170.47,155.2$ and 140 in environments $E_{1}$ to $E_{6}$ respectively

\section{Number of monopods /plant}

The overall range for this character in the six environments for the variety $\mathrm{H} 1098$ - I was $1.9-2.9$, H 1300 was $30.2-4.5$ and for the variety $\mathrm{H} 1316$ was 3.3 - 4. The mean number of monopods /plant of all the three varieties were $3.17,3.67,3.23,3.27,3.13$ and 2.87 in environments $\mathrm{E}_{1}$ to $\mathrm{E}_{6}$ respectively.

\section{Number of bolls/ plants}

The overall range for this character in the six environments for the variety $\mathrm{H} 1098$ - I was $17.4-29.2$, H 1300 was $16.1-25.3$ and for the variety $\mathrm{H} 1316$ was 13.6 - 25.1. The mean number of bolls /plant of all the three varieties were $21.5,17.7,15.7,26.5321 .33$ and 17.7 in environments $\mathrm{E}_{1}$ to $\mathrm{E}_{6}$ respectively.

\section{Boll weight (g)}

The overall range for this character in the six environments for the variety $\mathrm{H} 1098$ - I was $2.5-3.6, \mathrm{H} 1300$ was $2.2-3.5$ and for the variety $\mathrm{H} 1316$ was $2.2-3.3$.

The population mean of six environments was $3.23, \quad 2.7, \quad 2.37, \quad 3.47, \quad 2.83$ and 2.23 respectively. The mean number of bolls /plant of all the three varieties were $3.23,2.7,2.37$, $3.47,2.83$ and 2.23 in environments $E_{1}$ to $E_{6}$ respectively.

\section{Number of seeds/boll}

The overall range for this character in the six environments for the variety $\mathrm{H} 1098$ - I was 19.4 - 28.6, H 1300 was 18.4 - 25.9 and for the variety $\mathrm{H} 1316$ was 17.1 - 25. The mean number of seeds/boll of all the three varieties was $26.17,21.73,18.73,26.37,21.53$ and 21.17 in environments $E_{1}$ to $E_{6}$ respectively.

\section{Ginning out turn (\%)}

The overall range for this character in the six environments for the variety $\mathrm{H} 1098$ - I was 28 - 38, H 1300 was 23.9 - 37.4 and for the variety $\mathrm{H} 1316$ was $21.7-38$.

The mean ginning out turn (\%) of all the three varieties was $37.63,37.63,33.87,33.9731 .53$ and 34.52 in environments $E_{1}$ to $E_{6}$ respectively.

\section{Seed index (g)}

The overall range for this character in the six environments for the variety $\mathrm{H} 1098$ - I was $5.6-7.2, \mathrm{H} 1300$ was $5.7-6.9$ and for the variety $\mathrm{H} 1316$ was $5.9-6.9$. The mean seed index $(\mathrm{g})$ of all the three varieties was 6.97, $6.43,5.97,7,6.4$ and 6 in environments $E_{1}$ to $\mathrm{E}_{6}$ respectively.

\section{Lint index (g)}

The overall range for this character in the six environments for the variety $\mathrm{H} 1098$ - I was $2.5-4, \mathrm{H} 1300$ was $2.4-3.9$ and for the variety $\mathrm{H} 1316$ was $2.3-3.8$. The mean seed index $(\mathrm{g})$ of all the three varieties was 3.83 , $3.17,3,3.97,3.33$ and 2.4 in environments $E_{1}$ to $\mathrm{E}_{6}$ respectively. 


\section{A. Analysis of variance (ANOVA)}

Table.1 Environment wise analysis of variance of different characters

\begin{tabular}{|c|c|c|c|c|c|c|c|c|c|}
\hline \multirow{2}{*}{$\begin{array}{l}\text { Sr. } \\
\text { No }\end{array}$} & \multirow[t]{2}{*}{ Characters } & \multirow{2}{*}{$\begin{array}{l}\text { Sources of } \\
\text { Variance }\end{array}$} & \multirow[t]{2}{*}{ d.f } & \multicolumn{4}{|c|}{ Mean squares } & \multirow[b]{2}{*}{$\mathbf{E}_{5}$} & \multirow[b]{2}{*}{$\mathbf{E}_{6}$} \\
\hline & & & & $\mathbf{E}_{1}$ & $\mathbf{E}_{2}$ & $\mathbf{E}_{3}$ & $\mathbf{E}_{4}$ & & \\
\hline \multirow[t]{3}{*}{1} & \multirow{3}{*}{$\begin{array}{l}\text { Days To first } \\
\text { flower }\end{array}$} & Replication & 5 & 9.240 & 1.506 & 2.479 & 9.240 & 4.267 & 2.685 \\
\hline & & Genotypes & 2 & $59.209 *$ & $30.949 *$ & $32.445^{*}$ & $59.209 *$ & $71.341^{*}$ & $27.137^{*}$ \\
\hline & & Error & 10 & 11.324 & 5.090 & 1.184 & 11.324 & 9.021 & 3.183 \\
\hline \multirow[t]{3}{*}{2} & \multirow[t]{3}{*}{ Days to bursting } & Replication & 5 & 0.606 & 0.648 & 0.517 & 0.606 & 0.648 & 0.517 \\
\hline & & Genotypes & 2 & $11.949 *$ & $98.516^{*}$ & $27.727^{*}$ & $11.949^{*}$ & $15.182 *$ & $27.727 *$ \\
\hline & & Error & 10 & 0.488 & 0.364 & 0.420 & 0.488 & 0.364 & 0.420 \\
\hline \multirow[t]{3}{*}{3} & \multirow[t]{3}{*}{ Plant height $(\mathrm{cm})$} & Replication & 5 & 90.464 & 65.945 & 27.109 & 65.470 & 51.205 & 37.492 \\
\hline & & Genotypes & 2 & $1,057.040^{*}$ & $349.627 *$ & $182.220^{*}$ & $1,065.929 *$ & $251.407^{*}$ & $477.982 *$ \\
\hline & & Error & 10 & 28.968 & 46.688 & 13.561 & 26.452 & 48.652 & 40.406 \\
\hline \multirow[t]{3}{*}{4} & \multirow{3}{*}{$\begin{array}{l}\text { Number of } \\
\text { monopods /plant }\end{array}$} & Replication & 5 & 0.009 & 0.008 & 0.020 & 0.080 & 0.005 & 0.066 \\
\hline & & Genotypes & 2 & $0.349 *$ & $4.336^{*}$ & $0.807^{*}$ & $4.201 *$ & $4.160^{*}$ & $4.437 *$ \\
\hline & & Error & 10 & 0.013 & 0.026 & 0.007 & 0.031 & 0.035 & 0.023 \\
\hline \multirow[t]{3}{*}{5} & \multirow{3}{*}{$\begin{array}{l}\text { Number of bolls/ } \\
\text { plants }\end{array}$} & Replication & 5 & 1.191 & 1.205 & 2.096 & 0.425 & 1.849 & 0.840 \\
\hline & & Genotypes & 2 & $15.309 *$ & $2.991 *$ & $21.794 *$ & $32.204^{*}$ & $12.294 *$ & $13.051^{*}$ \\
\hline & & Error & 10 & 0.442 & 0.643 & 0.957 & 1.526 & 1.289 & 0.426 \\
\hline \multirow[t]{3}{*}{6} & \multirow[t]{3}{*}{ Boll weight(g) } & Replication & 5 & 0.002 & 0.010 & 0.007 & 0.138 & 0.008 & 0.006 \\
\hline & & Genotypes & 2 & $0.185^{*}$ & $0.304^{*}$ & $0.195^{*}$ & $0.167 *$ & $0.184^{*}$ & $0.352 *$ \\
\hline & & Error & 10 & 0.016 & 0.010 & 0.014 & 0.034 & 0.031 & 0.011 \\
\hline \multirow[t]{3}{*}{7} & \multirow{3}{*}{$\begin{array}{l}\text { Number } \\
\text { seeds/boll }\end{array}$} & Replication & 5 & 1.823 & 1.727 & 0.958 & 0.843 & 0.984 & 0.541 \\
\hline & & Genotypes & 2 & $26.327 *$ & $22.491 *$ & $19.517 *$ & $24.852^{*}$ & $22.862 *$ & $66.260 *$ \\
\hline & & Error & 10 & 1.307 & 0.904 & 0.574 & 0.758 & 0.540 & 0.351 \\
\hline \multirow[t]{3}{*}{8} & \multirow{3}{*}{$\begin{array}{l}\text { Ginning out turn } \\
(\%)\end{array}$} & Replication & 5 & 0.232 & 0.077 & 1.172 & 0.514 & 1.353 & 0.384 \\
\hline & & Genotypes & 2 & $0.735^{*}$ & $0.687 *$ & $3.852 *$ & $13.801^{*}$ & $2.996^{*}$ & $1.607^{*}$ \\
\hline & & Error & 10 & 0.095 & 0.130 & 0.874 & 0.301 & 0.688 & 0.295 \\
\hline \multirow[t]{3}{*}{9} & \multirow[t]{3}{*}{ Lint index (g) } & Replication & 5 & 0.005 & 0.020 & 0.085 & 0.024 & 0.013 & 0.013 \\
\hline & & Genotypes & 2 & $0.232 *$ & $0.667 *$ & $1.976^{*}$ & $0.229 *$ & $0.402 *$ & $0.065^{*}$ \\
\hline & & Error & 10 & 0.023 & 0.005 & 0.082 & 0.020 & 0.010 & 0.012 \\
\hline 10 & Seed index $(g)$ & Replication & 5 & 0.017 & 0.008 & 0.015 & 0.005 & 0.004 & 0.025 \\
\hline & & Genotypes & 2 & $0.082 *$ & $0.262 *$ & $0.554^{*}$ & $0.201 *$ & $0.305^{*}$ & $0.124^{*}$ \\
\hline & & Error & 10 & 0.010 & 0.006 & 0.005 & 0.024 & 0.008 & 0.024 \\
\hline 11 & yield/ & Replication & 5 & 1.572 & 2.850 & 0.424 & 2.937 & 0.396 & 0.516 \\
\hline & plant(g) & Genotypes & 2 & $43.641 *$ & $52.376^{*}$ & $62.162 *$ & $6.541 *$ & $5.932 *$ & $14.985^{*}$ \\
\hline & & Error & 10 & 0.961 & 2.533 & 0.434 & 0.699 & 1.182 & 0.624 \\
\hline 12 & Seed cotton & Replication & 5 & 17.783 & 11.940 & 0.582 & 5.620 & 5.753 & 11.069 \\
\hline & yield/ plant(g) & Genotypes & 2 & $207.402^{*}$ & $215.017 *$ & $83.962^{*}$ & $124.955^{*}$ & $152.602 *$ & $468.852 *$ \\
\hline & & Error & 10 & 6.490 & 11.379 & 6.140 & 4.672 & 14.303 & 9.673 \\
\hline
\end{tabular}

* Significant at $5 \%$ level 
Table.2 Mean value and range across environments for different characters

\begin{tabular}{|c|c|c|c|c|c|c|c|c|c|c|}
\hline S. No. & Characters & Genotypes & $\mathbf{E}_{1}$ & $\mathbf{E}_{2}$ & $\mathbf{E}_{3}$ & $\mathbf{E}_{4}$ & $\mathrm{E}_{5}$ & $\mathrm{E}_{6}$ & Average & Range \\
\hline \multirow[t]{4}{*}{1} & \multirow[t]{4}{*}{ Days To first flower } & H1098- I & 69.8 & 60 & 53.6 & 69.8 & 59.9 & 55.8 & 61.48 & $53.6-69.8$ \\
\hline & & H1300 & 64.5 & 58.9 & 50 & 64.5 & 57.2 & 52.5 & 57.93 & $50-64.5$ \\
\hline & & H1316 & 70.1 & 63.3 & 49.2 & 70.1 & 64 & 51.8 & 61.42 & $49.2-70.1$ \\
\hline & & Mean & 68.13 & 60.73 & 50.93 & 68.13 & 60.37 & 53.37 & 60.28 & \\
\hline \multirow[t]{4}{*}{2} & \multirow[t]{4}{*}{ Days to bursting } & H1098- I & 110.3 & 98.1 & 89.2 & 110.3 & 108.1 & 104.2 & 103.37 & $89.2-110.3$ \\
\hline & & H1300 & 112.9 & 106 & 93.5 & 112.9 & 111 & 108.5 & 107.41 & $93.5-112.9$ \\
\hline & & H1316 & 110.6 & 103.6 & 90.7 & 110.6 & 108.5 & 105.7 & 104.95 & $90.7-110.6$ \\
\hline & & Mean & 111.27 & 102.57 & 91.13 & 112.27 & 109.2 & 106.13 & 105.24 & \\
\hline \multirow[t]{4}{*}{3} & \multirow[t]{4}{*}{ Plant height $(\mathrm{cm})$} & H1098- I & 155.1 & 146.6 & 122.7 & 164.8 & 154.9 & 139.3 & 147.18 & $122.7-164.8$ \\
\hline & & H1300 & 150.9 & 138.9 & 111.7 & 160.9 & 148.9 & 131.5 & 140.47 & $111.7-160.9$ \\
\hline & & H1316 & 175.7 & 154.2 & 116.6 & 185.7 & 161.8 & 149.3 & 157.22 & $116.6-185.7$ \\
\hline & & Mean & 160.57 & 146.57 & 117 & 170.47 & 155.2 & 140 & 148.29 & \\
\hline \multirow[t]{4}{*}{4} & \multirow{3}{*}{$\begin{array}{l}\text { Number of monopods } \\
\text { /plant }\end{array}$} & H1098- I & 2.9 & 2.8 & 2.9 & 2.4 & 2.2 & 1.9 & 2.52 & $1.9-2.9$ \\
\hline & & H1300 & 3.3 & 4.5 & 3.2 & 3.4 & 3.4 & 3.2 & 3.5 & $3.2-4.5$ \\
\hline & & H1316 & 3.3 & 3.7 & 3.6 & 4 & 3.8 & 3.5 & 3.65 & $3.3-4$ \\
\hline & & Mean & 3.17 & 3.67 & 3.23 & 3.27 & 3.13 & 2.87 & 3.22 & \\
\hline \multirow[t]{4}{*}{5} & \multirow{4}{*}{$\begin{array}{l}\text { Number of bolls/ } \\
\text { plants }\end{array}$} & H1098- I & 20.6 & 18.5 & 17.4 & 29.2 & 22.9 & 19.3 & 21.32 & $17.4-29.2$ \\
\hline & & H1300 & 20.6 & 17.2 & 16.1 & 25.3 & 21 & 17.3 & 19.58 & $16.1-25.3$ \\
\hline & & H1316 & 23.3 & 17.4 & 13.6 & 25.1 & 20.1 & 16.5 & 19.33 & $13.6-25.1$ \\
\hline & & Mean & 21.5 & 17.7 & 15.7 & 26.53 & 21.33 & 17.7 & 20.08 & \\
\hline \multirow[t]{4}{*}{6} & \multirow[t]{4}{*}{ Boll weight(g) } & H1098- I & 3.4 & 2.9 & 2.5 & 3.6 & 3 & 2.5 & 2.98 & $2.5-3.6$ \\
\hline & & H1300 & 3.2 & 2.7 & 2.4 & 3.5 & 2.8 & 2.2 & 2.8 & $2.2-3.5$ \\
\hline & & H1316 & 3.1 & 2.5 & 2.2 & 3.3 & 2.7 & 2 & 2.63 & $2.2-3.3$ \\
\hline & & Mean & 3.23 & 2.7 & 2.37 & 3.47 & 2.83 & 2.23 & 2.80 & \\
\hline \multirow[t]{4}{*}{7} & \multirow[t]{4}{*}{ Number of seeds/boll } & H1098- I & 28.6 & 23.5 & 20.7 & 28.6 & 23.6 & 19.4 & 24.07 & $19.4-28.6$ \\
\hline & & H1300 & 25.1 & 22 & 18.4 & 25.9 & 21.2 & 19.1 & 21.95 & $18.4-25.9$ \\
\hline & & H1316 & 24.8 & 19.7 & 17.1 & 24.6 & 19.8 & 25 & 21.83 & $17.1-25$ \\
\hline & & Mean & 26.17 & 21.73 & 18.73 & 26.37 & 21.53 & 21.17 & 22.61 & \\
\hline \multirow[t]{4}{*}{$\overline{8}$} & \multirow[t]{4}{*}{ Ginning out turn (\%) } & H1098- I & 38 & 37.6 & 34.8 & 35.7 & 33.3 & 32 & 35.2 & $32-38.0$ \\
\hline & & H1300 & 37.4 & 37.3 & 33.5 & 33.2 & 32.4 & 31.6 & 34.23 & $31.6-37.4$ \\
\hline & & H1316 & 37.5 & 38 & 33.3 & 33 & 31.9 & 31 & 34.12 & 31-38 \\
\hline & & Mean & 37.63 & 37.63 & 33.87 & 33.97 & 32.53 & 31.53 & 34.52 & \\
\hline \multirow[t]{4}{*}{9} & \multirow[t]{4}{*}{ Seed index $(g)$} & H1098- I & 7.2 & 6.8 & 5.6 & 7.2 & 6.7 & 6 & 6.58 & $5.6-7.2$ \\
\hline & & H1300 & 6.9 & 6.4 & 5.7 & 6.9 & 6.3 & 6.1 & 6.38 & $5.7-6.9$ \\
\hline & & H1316 & 6.8 & 6.1 & 6.6 & 6.9 & 6.2 & 5.9 & 6.42 & $5.9-6.9$ \\
\hline & & Mean & 6.97 & 6.43 & 5.97 & 7 & 6.4 & 6 & 6.46 & \\
\hline \multirow[t]{4}{*}{10} & Lint index $(\mathrm{g})$ & H1098- I & 4 & 3.5 & 3.3 & 4.2 & 3.6 & 2.5 & 3.52 & $2.5-4$ \\
\hline & & H1300 & 3.8 & 3.3 & 3 & 3.9 & 3.3 & 2.4 & 3.28 & 2.4-3.9 \\
\hline & & H1316 & 3.7 & 3.1 & 2.7 & 3.8 & 3.1 & 2.3 & 3.12 & $2.3-3.8$ \\
\hline & & Mean & 3.83 & 3.17 & 3 & 3.97 & 3.33 & 2.4 & 3.31 & \\
\hline 11 & Lint yield/ plant(g) & H1098- I & 22.3 & 17.5 & 12.7 & 28.8 & 23.5 & 20.1 & 20.82 & $12.7-28.8$ \\
\hline & & H1300 & 25.3 & 21.5 & 18.3 & 30.6 & 22.7 & 18.3 & 22.78 & $18.3-30.6$ \\
\hline & & H1316 & 27.7 & 23.2 & 18.3 & 28.8 & 21.5 & 17 & 22.75 & $18.3-28.8$ \\
\hline & & Mean & 25.1 & 20.73 & 16.43 & 29.4 & 22.57 & 18.47 & 22.12 & \\
\hline 12 & Seed cotton yield/ & H1098- I & 58.6 & 46.6 & 29.2 & 79.9 & 68.9 & 40.7 & 53.98 & $29.2-79.9$ \\
\hline & plant $(g)$ & H1300 & 58.5 & 57 & 22.5 & 71.9 & 69.4 & 58.2 & 56.25 & $22.5-71.9$ \\
\hline & & H1316 & 68.7 & 56.8 & 23 & 72.2 & 60.4 & 51.7 & 55.47 & $23-72.2$ \\
\hline & & Mean & 61.93 & 53.47 & 24.9 & 74.67 & 66.23 & 50.2 & 55.23 & \\
\hline
\end{tabular}


Table.4 Estimates of stability parameters of individual genotypes

\begin{tabular}{|c|c|c|c|c|c|}
\hline Sr. No. & Characters & Genotypes & 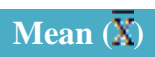 & $b_{i}$ & $\mathbf{S}_{\mathrm{di}}^{2}$ \\
\hline \multirow[t]{4}{*}{1} & \multirow[t]{4}{*}{ Days To first flower } & H1098- I & 61.48 & $0.94 *$ & $1.22^{*}$ \\
\hline & & H1300 & 57.93 & $0.83 *$ & -0.84 \\
\hline & & H1316 & 61.42 & $1.23 *$ & $1.73 *$ \\
\hline & & Population mean & 60.28 & & \\
\hline \multirow[t]{4}{*}{2} & \multirow{4}{*}{ Days to bursting } & H1098- I & 103.37 & $1.07 *$ & $1.77 *$ \\
\hline & & H1300 & 107.41 & $0.95^{*}$ & 0.40 \\
\hline & & H1316 & 104.95 & $0.97 *$ & 0.39 \\
\hline & & Population mean & 105.24 & & \\
\hline \multirow[t]{4}{*}{3} & \multirow[t]{4}{*}{ Plant height $(\mathrm{cm})$} & H1098- I & 147.18 & $0.79 *$ & -2.98 \\
\hline & & H1300 & 140.47 & $0.93 *$ & -4.00 \\
\hline & & H1316 & 157.22 & $1.28^{*}$ & 2.75 \\
\hline & & Population mean & 148.29 & & \\
\hline \multirow[t]{4}{*}{4} & \multirow[t]{4}{*}{ Number of monopods /plant } & H1098- I & 2.52 & $1.06^{*}$ & 0.12 \\
\hline & & H1300 & 3.50 & $1.74 *$ & 0.06 \\
\hline & & H1316 & 3.65 & 0.19 & 0.07 \\
\hline & & Population mean & 3.22 & & \\
\hline \multirow[t]{4}{*}{5} & \multirow[t]{4}{*}{ Number of bolls/ plants } & H1098- I & 21.32 & 1.06 & $1.62 *$ \\
\hline & & H1300 & 19.58 & $0.88^{*}$ & -0.04 \\
\hline & & H1316 & 19.33 & $1.06^{*}$ & $2.19 *$ \\
\hline & & & 20.08 & & \\
\hline \multirow{4}{*}{6} & \multirow[t]{4}{*}{ Boll weight(g) } & H1098- I & 2.98 & $0.97 *$ & 0.001 \\
\hline & & H1300 & 2.80 & 0.98 & 0.002 \\
\hline & & H1316 & 2.63 & $1.05^{*}$ & 0.001 \\
\hline & & Population mean & 2.80 & & \\
\hline \multirow[t]{4}{*}{7} & \multirow[t]{4}{*}{ Number of seeds/boll } & H1098- I & 24.07 & $1.18^{*}$ & 2.68 \\
\hline & & H1300 & 21.95 & $0.97 *$ & 0.67 \\
\hline & & H1316 & 21.83 & $0.85^{*}$ & 6.09 \\
\hline & & Population mean & 22.61 & & \\
\hline \multirow[t]{4}{*}{8} & \multirow[t]{4}{*}{ Ginning out turn (\%) } & H1098- I & 35.20 & $0.89 *$ & 0.30 \\
\hline & & H1300 & 34.23 & $0.97 *$ & 0.01 \\
\hline & & H1316 & 34.12 & $1.14 *$ & 0.06 \\
\hline & & Population mean & 34.52 & & \\
\hline \multirow[t]{4}{*}{9} & \multirow[t]{4}{*}{ Seed index $(\mathrm{g})$} & H1098- I & 6.58 & $1.38^{*}$ & 0.06 \\
\hline & & H1300 & 6.38 & 1.01 & 0.01 \\
\hline & & H1316 & 6.42 & $0.61 *$ & 0.12 \\
\hline & & Population mean & 6.46 & & \\
\hline \multirow[t]{4}{*}{10} & \multirow[t]{4}{*}{ Lint index $(\mathrm{g})$} & H1098- I & 3.52 & $0.98^{*}$ & 0.001 \\
\hline & & H1300 & 3.28 & $1.00^{*}$ & 0.000 \\
\hline & & H1316 & 3.12 & $1.02 *$ & 0.001 \\
\hline & & Population mean & 3.31 & & \\
\hline \multirow[t]{4}{*}{11} & Lint yield/ plant(g) & H1098- I & 20.82 & $1.07 *$ & 6.23 \\
\hline & & H1300 & 22.78 & $0.98^{*}$ & 0.56 \\
\hline & & H1316 & 22.75 & 0.95 & 4.17 \\
\hline & & Population mean & 22.12 & & \\
\hline 12 & Seed cotton yield/ plant $(\mathrm{g})$ & H1098- I & 53.98 & $1.03 *$ & 0.46 \\
\hline & & H1300 & 56.25 & $0.99 *$ & 0.24 \\
\hline & & H1316 & 55.47 & 1.00 & 0.20 \\
\hline & & Population mean & 55.23 & & \\
\hline * - Signif & $\begin{array}{l}t \text { at } 5 \% \text { level } \\
\text { nt at } 1 \% \text { level }\end{array}$ & $\begin{array}{l}\text { egression coeffic } \\
\text { Deviation from } 1\end{array}$ & & & \\
\hline
\end{tabular}


Table.3 Analysis of variance for stability parameter of various traits (Eberhert and Russell, 1966)

\begin{tabular}{|c|c|c|c|c|c|c|c|c|c|c|c|c|c|c|}
\hline $\begin{array}{l}\text { Sr. } \\
\text { No }\end{array}$ & $\begin{array}{l}\text { Sources of } \\
\text { variance }\end{array}$ & d.f & $\begin{array}{l}\text { Days to } \\
\text { first } \\
\text { flower }\end{array}$ & $\begin{array}{c}\text { Days to } \\
\text { boll } \\
\text { bursting }\end{array}$ & $\begin{array}{c}\text { Plant } \\
\text { height }(\mathrm{cm})\end{array}$ & $\begin{array}{l}\text { Number of } \\
\text { monopods } \\
\text { per plant }\end{array}$ & $\begin{array}{l}\text { Number } \\
\text { of bolls } \\
\text { per plant }\end{array}$ & $\begin{array}{c}\text { Boll } \\
\text { weight } \\
\text { (g) }\end{array}$ & $\begin{array}{l}\text { Number } \\
\text { of seeds } \\
\text { per boll }\end{array}$ & $\begin{array}{c}\text { Ginning } \\
\text { out turn } \\
(\%)\end{array}$ & $\begin{array}{l}\text { Lint } \\
\text { index } \\
(\mathrm{g})\end{array}$ & $\begin{array}{l}\text { Seed } \\
\text { index } \\
(\mathbf{g})\end{array}$ & $\begin{array}{l}\text { Lint cotton } \\
\text { yield/plant } \\
\text { (g) }\end{array}$ & $\begin{array}{c}\text { Seed } \\
\text { cotton } \\
\text { yield/plant } \\
\text { (g) }\end{array}$ \\
\hline 1 & $\begin{array}{c}\text { Genoty } \\
\text { pes }\end{array}$ & 2 & $155.57 *$ & $25.43 *$ & $426.05^{*}$ & $2.34 *$ & $7.01 *$ & $0.22 *$ & $9.51 *$ & $2.29 *$ & $0.06 *$ & $0.23 *$ & $7.55^{*}$ & $8.04^{*}$ \\
\hline 2 & $\begin{array}{c}\text { Env. + } \\
\text { (G X E) }\end{array}$ & $\begin{array}{l}1 \\
5\end{array}$ & $54.79 *$ & $59.89 *$ & $366.10^{*}$ & $0.16^{*}$ & $16.26^{*}$ & $0.22 *$ & $11.88 *$ & $6.82 *$ & $0.27 *$ & $0.34^{*}$ & $25.04 *$ & $324.94 *$ \\
\hline 3 & $\begin{array}{c}\text { Env. } \\
\text { (linear) }\end{array}$ & 1 & $777.86 *$ & $884.81 *$ & $5,215.47 *$ & $1.01 *$ & $225.39 *$ & $3.28 *$ & $136.48 *$ & $98.91 *$ & $3.03 *$ & $5.02 *$ & $328.89 *$ & $4,472.54 *$ \\
\hline 4 & $\begin{array}{c}\mathrm{G} \times \mathrm{E} \\
\text { (linear) }\end{array}$ & 2 & $10.90 *$ & $1.20 *$ & $112.31 *$ & 0.20 & $0.83 *$ & 0.02 & $1.26 *$ & $0.51 *$ & 0.15 & 0.01 & $2.41 *$ & $3.74 *$ \\
\hline 5 & $\begin{array}{c}\text { Pooled } \\
\text { deviatio } \\
n\end{array}$ & $\begin{array}{l}1 \\
2\end{array}$ & 1.84 & $0.92 *$ & $4.28 *$ & 0.09 & $1.41 *$ & 0.01 & $3.27 *$ & $0.19 *$ & $0.07 *$ & 0.04 & $3.83 *$ & $33.34 *$ \\
\hline 6 & $\begin{array}{l}\text { Pooled } \\
\text { error }\end{array}$ & $\begin{array}{l}6 \\
0\end{array}$ & 6.85 & 0.42 & 34.12 & 0.02 & 0.88 & 0.02 & 0.74 & 0.40 & 0.03 & 0.01 & 1.07 & 8.78 \\
\hline
\end{tabular}

* - Significant at $5 \%$ level

Table.5 Environmental indices for different characters in upland cotton in different environments expressed as deviation from grand mean (Environmental additive effects)

\begin{tabular}{|c|c|c|c|c|c|c|c|c|}
\hline \multirow{2}{*}{$\begin{array}{l}\text { Sr. } \\
\text { No. }\end{array}$} & \multirow[t]{2}{*}{ Characters } & \multicolumn{6}{|c|}{ Environmental index } & \multirow[t]{2}{*}{ Grand mean } \\
\hline & & $\mathbf{E}_{1}$ & $\mathbf{E}_{2}$ & $\mathbf{E}_{3}$ & $\mathbf{E}_{4}$ & $\mathbf{E}_{5}$ & $\mathbf{E}_{6}$ & \\
\hline 1. & Days to first flower & 7.85 & 0.45 & -9.35 & 7.85 & 7.85 & -6.91 & 60.28 \\
\hline 2 & Days to boll bursting & 7.03 & 2.67 & -14.11 & 7.03 & 3.96 & 0.89 & 105.24 \\
\hline 3 & Plant height $(\mathrm{cm})$ & 12.28 & -1.72 & -31.29 & 22.18 & 6.91 & -8.29 & 148.29 \\
\hline 4 & Number of monopods per plant & -0.05 & 0.45 & 0.01 & 0.05 & -0.09 & -0.35 & 3.22 \\
\hline 5 & Number of bolls per plant & 1.42 & -2.38 & -4.38 & 6.45 & 1.25 & -2.38 & 20.08 \\
\hline 6 & Boll weight $(\mathrm{g})$ & 0.43 & -0.1 & -0.43 & 0.67 & 0.03 & -0.57 & 2.80 \\
\hline 7 & Number of seeds per boll & 3.56 & -0.88 & -3.88 & 3.76 & -1.08 & -1.44 & 22.61 \\
\hline 8 & Ginning out turn $(\%)$ & 4.76 & 4.76 & -0.65 & -0.55 & -1.99 & -2.99 & 34.52 \\
\hline 9 & Seed index $(\mathrm{g})$ & 0.51 & -0.03 & -0.49 & 0.54 & -0.04 & -0.46 & 6.46 \\
\hline 10 & Lint index $(\mathrm{g})$ & 0.52 & -0.14 & -0.31 & 0.66 & 0.02 & -0.91 & 3.31 \\
\hline 11 & Lint yield per plant $(\mathrm{g})$ & 2.98 & -1.39 & -5.69 & 7.28 & 0.45 & -3.65 & 22.12 \\
\hline 12 & Seed cotton yield per plant (g) & 6.7 & -1.76 & -30.33 & 19.44 & 5.4 & -5.03 & 55.23 \\
\hline
\end{tabular}

\section{Lint yield/ plant (g)}

The overall range for this character in the six environments for the variety $\mathrm{H} 1098$ - I was12.7 - 28.8, H 1300 was 18.3 - 30.6 and for the variety $\mathrm{H} 1316$ was 18.3 - 28.8. The mean lint yield / plant $(\mathrm{g})$ of all the three varieties were $25.1,20.73,16.43,29.4,22.57$ and 18.47 in environments $\mathrm{E} 1$ to $\mathrm{E} 6$ respectively.

\section{Seed cotton yield/ plant $(g)$}

The overall range for this character in the six environments for the variety $\mathrm{H} 1098$ - I was 29.2 - 79.9, H1300 was $22.5-71.9$ and for the variety H1316 was 23 - 72.2. The mean seed cotton yield / plant $(\mathrm{g})$ of all the three varieties were $61.93,53.47,24.9,74.67,66.23$ and 50.2 in environments $\mathrm{E}_{1}$ to $\mathrm{E}_{6}$ respectively. 
Environmental mean squares were also highly significant for all the characters which indicated that the environments chosen in the study were highly variable (Table 3 ). Mean squares due to environment (linear) and linear interaction genotypes $x$ environment were significantly for most of the traits studied. This indicated that differences in environments (sowing dates and years) will generate disparities on cultivar responses; while the later effect indicates that there are genetic divergences among cultivars taking into account their responses to variation in environmental conditions. The main cause of the differences among genotypes in their yield stability traits was the wide occurrence of $\mathrm{G} x$ E interaction. Similar results were found by (Abdallah et al., 2011; Dewdar, 2013 and Kavithamani et al., 2013).

The variety H 1098 - I had high mean and bi $>1$ for number of monopods / plant (2.52), number of seeds / boll (24.07), seed index (6.58), lint yield / plant (3.52) and for seed cotton yield / plant (53.98) indicated its suitability for better environment (Table 4). The variety $\mathrm{H} 1300$ was found to be most stable and average response to wider range of environments as they had higher mean, regression coefficient near to unity and least deviation from regression number of monopods / plant (3.65), lint yield / plant (22.75) and seed cotton yield / plant (55.47). The variety $\mathrm{H} 1316$ had high mean and bi $>1$ for plant height (157.22), boll weight (2.63), ginning outturn (344.12) and lint index (3.12) indicating its suitability for better environment. While it had $b<1$ for days to boll bursting (104.95), number of seed / boll (21.83) and seed index (6.42) indicated its more adoptability to poor environment. These results were in agreement with findings of Patil and Patel, 2010.

The estimates of environmental additive effects (Ij) are presented in table 5. Perusal of table revealed that environment 4 i.e. early sown of 2016 was best for seed cotton yield per plant (g). It was found best for all the traits under study. Environment 1 was best for all the traits under study except for number of monopods/ plant whereas environment 2 was found to be favourable for all the traits except for the number of monopods per plant. Environment 3 was poor for most of the traits studied except for the number of monopods per plant. Environment 5 was found to be favourable for to first flower, days to boll bursting, plant height, number of bolls per plant, boll weight, lint index, lint yield per plant and seed cotton yield per plant. Environment 6 was poor for most of the traits studied except for the days to boll bursting.

Table 5 clearly showed that during the year 2015 environmental index values were much higher than 2016 in their respective environments for ginning out turn trait mainly due to very poor seed development during kharif, 2015 and ultimately resulted in reduced seed index and poor seed cotton yield also. The environment 3 i.e. late sowing of 2015 was the poorest environment for all the traits. When we compare the different environments of a particular year, it clearly showed that early sowing was most favorable, followed by normal sowing and late sowing as it resulted in poorest performance for most of the traits and similar observations were during the year 2016. Among the years, 2016 was the favorable one. Similar pattern of results were observed by Sunayana et al., 2018.

\section{References}

Abdallah, T.A., Abd El-Moula, M.A., ElKoomy, M.B.A., Mostafa, M.A., Khalil, M.A.G. (2011). Genotype x environment interaction and stability parameters for grain yield in some 
promising maize hybrids. Egypt. J. Plant Breed. 15: 61-70.

Campbell, B.T., P.W. Lubbers, C.E., Bowman, D.T., Meredith, W.R., Johnson, J., Bridges, D. and Jones, D.C. (2012). Dissecting genotype $\times$ environment interactions and trait correlations present in the pee dee cotton germplasm collection following seventy years of plant breeding. Crop Sci. 52(2): 690-699.

Dahiphale, K. D. And Deshmukh, J. D. (2018). Genetic variability, correlation and path coefficient analysis for yield and its attributing traits in cotton (Gossypium hirsutum L.) J. Cotton Res. Dev.32 (1): 38-46.

Dewdar, M.D.H. (2013). Stability analysis and genotype $\mathrm{x}$ environment interactions of some Egyptian cotton cultivars cultivated. African J. Ag. Res. 8(41): 5156-5160.

Eberhart, S.A. and Russell, W.A. (1966). Stability parameters for comparing varieties. Crop Sci. 6:36-40.

Gomez, K.A. and Gomez, A.A. (1984). Statistical Procedures for Agricultural
Research (2nd ed.). John Wiley \& Sons Inc., New York. USA.

ICAR-All India Coordinated Research Project on Cotton Annual Report 2016-2017.

Kavithamani, D., Amala, Balu, P. and Rajarathinam, S. (2013). Assessment of genetic diversity in Gossypium barbadense. J.Cotton Res.Dev. 27: 2023.

Killi, F. and Harem, E. (2006). Genotype $\times$ environment interaction and stability analysis of cotton yield in Aegean region of Turkey. J. Environ. Biol. 27(2): 427-430.

Patil, H.E. and Patel, K.G. (2010). Stability analysis of seed cotton yield and its components of released $\mathrm{Bt}$ cotton hybrids of Gujarat state. J. Cotton Res. Dev. 24: 17-22.

Sunayana, Sangwan, R.S., Nimbal, S., Mor, V.S. And Pinki (2018).Impact of genotype $\times$ environment interaction and stability for seed cotton yield and its component traits in Asiatic cotton (Gossypium arboreum L.) J. Cotton Res. Dev. 32 (1): 23-29.

\section{How to cite this article:}

Pinki, S.S. Siwach, R.S. Sangwan, Sombir Singh, V.S. Mor, Shiwani Mandhania, Sunayana and Neha Rohila. 2018. Stability Analysis for Seed Cotton Yield and Its Components in Upland Cotton (Gossypium hirsutum L.). Int.J.Curr.Microbiol.App.Sci. 7(04): 1630-1638. doi: https://doi.org/10.20546/ijcmas.2018.704.184 